\title{
Chirurgische Gastroenterologie
}

\author{
Klinische Angiologie \\ Herausgeber: H. Rieger, W. Schoop \\ unter Mitarbeit von A. L. Strauss, A. Schepler, G. Driessen \\ Springer, Berlin 1998 \\ 1420 S., 745 z. T. farb. Abb., 264 Tab., DM 368,- \\ ISBN 3-540-50899-6
}

Die Angiologie wurde 1992 nicht zuletzt als jüngstes Schwerpunktfach in der Inneren Medizin etabliert, da Prävalenz und Inzidenz angiologischer Leiden die Größenordnung einer Volkskrankheit haben und deswegen entsprechende Strukturen zur Versorgung Gefäßkranker aufgebaut werden müssen. Auffällig ist dabei, daß in Deutschland 1995 erst wenige weitergebildete Angiologen ca. 700 Gefäßchirurgen gegenübergestellt werden können. Dem Ziel der Weiterverbreitung und Institutionalisierung der Angiologie ist dieses Buch gewidmet, das eine Brücke zwischen Lehrbuch und Nachschlagwerk sein will. Es ist in 6 Bereiche eingeteilt und beginnt mit den peripheren Arterienerkrankungen und arteriellen Durchblutungsstörungen, setzt sich mit der Erörterung der Mikrozirkulationsstörungen, der peripheren Venenerkrankungen und venösen Rückflußstörungen, der peripheren Lymphgefäßerkrankungen und Lymphabflußstörungen, der zerebralen Durchblutungsstörungen und vaskulären Neo- und peripheren Angiodysplasien fort. Zum Schluß folgt ein ausführliches Sachverzeichnis. An diesem Buch sind über 50 angiologisch, radiologisch und chirurgisch ausgewiesene Mitarbeiter, größtenteils aus Deutschland, vereinzelt aus dem Ausland, beteiligt. Es spannt einen Bogen von der pathologischen Anatomie bis zu den kongenitalen peripheren Angiodysplasien und arteriovenösen Fisteln. Trotz der vielen Mitarbeiter zeichnet sich ein roter Faden durch das Buch, der durch die Herausgeber, den interventionellen Angiologen Rieger und den konventionellen Angiologen Schoop, der als einer der Väter der Angiologie bezeichnet werden kann, garantiert ist. Es enthält zahlreiche, z. T. farbige Abbildungen, Tabellen und nach jedem Kapitel ein ausführliches Literaturverzeichnis. Dem Kapitel vorangestellt ist ein ausführliches Inhaltsverzeichnis. Insgesamt füllt das Buch eine Lücke bei den Bemühungen zum Erlangen eines umfassenden Wissens über die Angiologie und wird daher nicht nur den Krankenhausbibliotheken, sondern auch allen Internisten, internistisch und angiologisch interessierten Allgemeinmedizinern und natürlich den Nachbarwissenschaften wie Gefäßchirurgie und Radiologie zum eifrigen Studium oder zur Orientierung ohne Einschränkung empfohlen. Der Preis ist angemessen.

K.-J. Paquet, Hannover

\author{
Hygienemaßnahmen in Krankenhaus und Praxis \\ Ein Ratgeber für Ärzte und Pflegepersonal \\ Herausgeber: V. Hingst, H.-G. Sonntag \\ Wissenschaftliche Verlagsgesellschaft mbH, Stuttgart 1997 \\ 6 Abb., 19 Tab., DM 38,- \\ ISBN 3-8047-1460-9
}

Die Hygiene hat mit der Umsetzung des Gesundheitsstrukturgesetzes in allen Krankenhäusern ab 1996 und der damit verbundenen Neustrukturierung der Finanzierung des Krankenhauswesens in Deutschland als Disziplin mit wesentlichen Elementen der Qualitätssicherung einen neuen Stellenwert erhalten. Es ist daher verständlich, daß neue Anforderungen für Informationsmöglichkeiten auf dem Gebiet der Krankenhaushygiene gestellt werden, die bisher bis auf wenige Einzelfälle lediglich über Journale abgedeckt wurden. Deswegen haben sich die Herausgeber mit dem vorliegenden Buch das Ziel gesetzt, ein kurzes Nachschlagewerk zu praktischen Belangen der Krankenhaushygiene zu schaffen, das sowohl für die Hygienefachkraft, den Hygienebeauftragten als auch allen ärztlichen Mitarbeitern und medizinischem Assistenzpersonal für den täglichen Gebrauch als Taschenbuch in der Kitteltasche ständig zur Verfügung steht. Auf diese Weise können sie bei auftretenden krankenhaushygienischen Problemen ihr Wissen auffrischen oder sich in ihrer Ansicht bestätigen lassen. Das Buch beginnt mit der Darstellung der Epidemiologie und mikrobiologischen Diagnostik von Krankenhausinfektionen, setzt sich mit der Darstellung der Grundlagen der Prävention von Infektionskrankheiten, ihrer allgemeinen und speziellen Prophylaxe, deren Integration in den Funktionsablauf und die Darstellung der Bereiche der Verund Entsorgung fort. Daran schließt sich die Organisation praktischer Infektionsprophylaxe zur Qualitätssicherung und die Darstellung der gesetzlichen und normativen Grundlagen der Krankenhaushygiene an. Es folgt ein Anhang mit Darstellungen der Regelung über Postversand von medizinischem und biologischem Untersuchungsgut, der mikrobiologischen Diagnostik, der Empfehlung des Robert-Koch-Instituts, des Bundesinstituts für Infektionskrankheiten und nichtübertragbare Krankheiten, der Geschäftsordnung für die Hygienekommission von Krankenhäusern und der Kommission für Krankenhaushygiene und Infektionsprävention sowie Adressen von Weiterbildungsstätten. Den Schluß bildet ein relativ sparsames Sachregister. Dieses Taschenbuch erfüllt seinen Zweck in jeder Hinsicht und kann daher zum eifrigen Studium bzw. zum Nachsehen für die angegebene Zielgruppe sehr empfohlen werden. Sein Preis ist günstig.

K.-J. Paquet, Hannover

\section{KARGER}

Fax +497614520714

E-mail kargergmbh@aol.com

www.karger.com (c) 1999 S. Karger GmbH, Freiburg

Accessible online at:

http://BioMedNet.com/karger 


\section{Hepato-Pancreato-Biliary Diseases - State of the Art}

Editors: C. E. Broelsch, J. R. Izbicki, K. A. Gawad, C. Bloechle

Johann Ambrosius Barth, Heidelberg 1998

3 CD-ROM (MAC/Windows) with Booklet, DM 98,ISBN 3-35335-00540-6

Anfang Juni 1997 fand in Hamburg der Europäische Kongreß der Internationalen Vereinigung für Erkrankungen von Leber, Bauchspeicheldrüse und Gallenwege statt. 600 Teilnehmern aus 45 Ländern wurden hervorragende Beiträge im Sinne eines «State of the Art» der Leberverpflanzung, der chronischen Pankreatitis, der entzündlichen Darmerkrankungen in Zusammenhang mit der sklerosierenden Cholangitis, von Klatskin-Tumoren und Lebermalignomen, des Pankreaskarzinoms und gutartiger Lebererkrankungen, der akuten Pankreatitis, der laparoskopischen Chirurgie und endoskopischen Therapie von malignen Erkrankungen von Leber, Bauchspeicheldrüse und Gallenwege sowie der Behandlung von Gallengangsteinen geboten. Diese wurden ergänzt durch Übersichtsreferate über Ethik, Standard der Lebensqualität und Standards des chirurgischen Trainings sowie zahlreiche Poster und Videofilme. Die wesentlichen «State of the Art»-Darbietungen sind auf der vorliegenden CD-ROM dem Ende August des gleichen Jahres verstorbenen Professor Dr. Dr. h. c. Rudolf Pichlmayr, dem Ehrenpräsidenten und einem Pionier in der hepatobiliären und Pankreaschirurgie gewidmet. Erfaßt wurden auf den Beiträgen Photos der Referenten und die größtenteils in Farbe vorgestellten Diapositive. Sie enthalten wichtige Informationen, so daß ihr Studium für die Aktualisierung des Wissens auf den o. a. Gebieten allen Viszeral- und Transplantationschirurgen sowie Gastroenterologen, Hepatologen und Radiologen empfohlen werden kann. Die Präsentation ist gelungen.

K. J. Paquet, Hannover

\section{Topographische Anatomie des Menschen}

Herausgeber: G.-H. Schumacher, G. Aumüller

6. Aufl.

Gustav Fischer, Stuttgart 1994, UTB für Wissenschaft

DM 39,80

Taschenbuch UTB-Bestell-Nr. ISBN 3-8252-0629-7

Dieses Taschenbuch erscheint bereits in der 6. Auflage, die hinsichtlich ihres Bildmaterials erneut überarbeitet wurde. Die Topographische Anatomie ist eine Synthese der systematischen Anatomie. Sie lehrt den Aufbau von Regionen und Körperhöhlen nach den räumlichen Beziehungen der Organe oder verschiedener Organsysteme zueinander. Im vorliegenden Taschenbuch wurde aus der großen Fülle anatomischer Fakten eine Auswahl getroffen, die den Bedürfnissen des angehenden und des praktisch tätigen Arztes gerecht werden soll. Die einzelnen Körperregionen werden von oben nach unten, d. h. vom Kopf über Hals, Brust, Bauch, Becken, Rücken, Bein und Arm, abgehandelt. Sie schließen mit der Besprechung der Knochenkerne, akzessorischer Skelettelemente, der Zusammenstellung der im Text genannten Eigennamen, Quellennachweise der Abbildungen, Fragen zum Selbststudium und einem Sachregister ab. Die teilweise 3farbigen Abbildungen, die etwas reichhaltiger vorhanden sein könnten, sind illustrativ und gut zu verstehen. Der Student wird besonders dankbar die Fragen zum Selbststudium annehmen. Der Umfang des Sachverzeichnisses ist ausreichend. Das Buch kann mit wenigen Einschränkungen dem Medizinstudenten und angehenden sowie praktisch tätigen Arzt zum Studium empfohlen werden. Sein Preis ist günstig.

\section{K.-J. Paquet, Hannover}

\section{Begutachtung der Haltungs- und Bewegungsorgane}

Herausgeber: G. Rompe, A. Erlenkämper

3., überarb. und erw. Auflage

Thieme, Stuttgart-New York 1998

29 Abb., 13 Tab., DM 198,-

ISBN 3-13-559203-0

Seit der 2. Auflage dieses Buches, die 1992 erschienen ist, hat das Sozialrecht umfangreiche Änderungen erfahren; darüber hinaus haben sich zahlreiche medizinisch-wissenschaftliche Auffassungen zu Einzelfragen der Begutachtung fortentwickelt. Eine Neuauflage des Werkes war somit notwendig. Eingeschlossen wurden die gesetzliche Pflegeversicherung und die gesetzliche Unfallversicherung wegen ihrer Neuordnung und Übernahme in das Sozialgesetzbuch. Neuere Änderungen der Rechtsprechung und Rechtslehre, insbesondere zur Beurteilung ursächlicher Zusammenhänge in wichtigen Einzelfragen, wurden in dem Kapitel «Juristische Grundlagen» neu aufgenommen. Der medizinische Bereich wurde um die Kapitel Osteoporose, Knochentumoren, entzündlich-rheumatische Gelenkerkrankung, Tauglichkeit von Fahrerlaubnisbewerbern und der bandscheibenbedingten Erkrankungen der Wirbelsäule erweitert. Der tabellarische Teil der Bewertungsvorschläge für die verschiedenen Gutachtendisziplinen wurde übersichtlich an das Ende des Bandes verlagert und durch eine Synopse aktueller Begutachtungsvorschläge im Rahmen der Gliedertaxe der privaten Unfallversicherung erweitert. Das Autorenteam, ein Vorsitzender Richter am Sozialgericht und ein Ordinarius für Chirurgie, hat sich bei der Abhandlung der juristischen Grundlagen und der medizinischen Aspekte bei der Begutachtung der Haltungs- und Bewegungsorgane sehr bewährt. Eingeschlossen ist auch die Beurteilung der Wehrdienstfähigkeit, von Hilfen nach dem Bundessozialgericht und bei Berufserkrankungen. Am Anfang werden die Abkürzungen auf 2 Seiten erläutert. Angenehm ist ein Anfangskapitel, das die zentralen Rechtsbegriffe erläutert und behandelt, die dem Arzt immer wieder Schwierigkeiten bereiten. Ihm folgt ein weiteres Kapitel, das sich mit dem ursächlichen Zusammenhang befaßt und die Ärzte beim kausalen Denken unterstützt. Das relativ trockene Gebiet wird in einem angenehmen Schriftbild dargeboten und leicht verständlich vermittelt. Mehr Abbildungen und Tabellen hätten dieses Ziel des illustrativen Verständnisses noch weiter verbessert. Jedes Kapitel hat ein kurzes, aktuelles Literaturverzeichnis, wobei das Sachverzeichnis am Ende etwas knapp geraten ist. Dennoch kann das Buch allen mit der Begutachtung der Haltungsund Bewegungsorgane befaßten Orthopäden und Chirurgen und vereinzelt auch Allgemeinmediziner zum Studium empfohlen werden. Sein Preis ist angemessen.

K.-J. Paquet, Hannover 